\title{
MUDAS DE LEGUMINOSAS ARBÓREAS INTRODUZIDAS EM PASTAGEM DE TIFTON-85 COM ANIMAIS
}

\author{
YOUNG LEGUMINOUS TREE INTRODUCED INTO TIFTON-85 PASTURES \\ WITHANIMALS
}

Dias, P.F. ${ }^{1}$, S.M. Souto ${ }^{2}$ e A.A. Franco ${ }^{2}$ 1Estação Experimental de Seropédica da PESAGRO.BR 465, km 7. CEP 23890-000. Seropédica-RJ. Brasil.
pfrancisco@hotmail.com.br
EmbrapaAgrobiologia.BR465,km7.CEP23851-970.Seropédica-RJ.Brasil.smsouto@cnpab.embrapa.br;
avilio@cnpab.embrapa.br

\section{PaLAVRAS CHAVES ADICIONAIS}

Aceitabilidade. Brotos. Capim Bermuda. Gliricidia sepium. Mimosa tenuiflora.

\section{RESUMO}

Em pastagem de capim bermuda cv. Tifton-85 foi avaliado o desempenho de mudas de 16 espécies de leguminosas arbóreas, sem proteção e na presença de animais, em três épocas diferentes do ano, no município de Seropédica-RJ. Foram selecionadas nove variáveis relacionadas ao comprimento e número de brotos das mudas, antes e após o pastejo com 30 novilhas mestiças leiteiras. Diferenças entre as médias dos tratamentos para cada variável, foram calculadas por meio de intervalos de confiança de Bonferroni, mostraram que jurema preta (Mimosa tenuiflora) apresentou a menor diferença no comprimento e número de brotos após o pastejo. Os resultados relacionados às variáveis após o pastejo para a jurema preta confirmam a sua recomendação para a região, como a leguminosa arbórea com maior probabilidade de sucesso, para introdução na pastagem, sem a proteção das mudas e na presença do gado.

\section{SUMMARY}

The introduction of unprotected young plants of 16 leguminous tree species into bermuda grass cv. Tifton-85 pastures under grazing was evaluated in four different moments of the year at the Seropedica Municipality, RJ. Nine variables related to length and number of sproutings were used for the evaluations before and after animal

Recibido: 1-8-07. Aceptado: 17-1-08.

\section{Additional KeYWORDS}

Acceptability. Bermuda grass. Gliricídia sepium. Mimosa tenuiflora. Sproutings.

grazing. Difference among treatments means for each variable, calculated by Bonferroni confidence intervals, revealed that jurema preta (Mimosa tenuiflora) presented the difference smallest on length and number of sproutings after grazing. The results confirm the recommendation of jurema preta as the tree legume with the highest chances of successful growth for studied region, always the introduction into the pastures is to be made without grazing protection.

\section{INTRODUÇÃO}

Espécies arbóreas são necessárias para melhorar a produção, qualidade e a sustentabilidade das pastagens (Ibrahim et al., 2001; Costa et al., 2005), acumular quantidades substanciais de carbono (Kanninen, 2001; McAdam et al., 2005) e aumentar a biodiversidade em pastagens (Naranjo, 2000; McAdam et al., 2005). Esses benefícios são maiores no caso de leguminosas arbóreas que possuem a capacidade de fixar o nitrogênio do ar (Dias, 2005).

Segundo Andrade et al. (2002), entre as razões de pecuaristas considerarem indesejável a presença de árvore nas pastagens, está a dificuldade para introdução de suas 


\section{DIAS, SOUTO E FRANCO}

mudas nos pastos. Montoya e Baggio (1991), estudando a viabilidade econômica da introdução de mudas florestais para sombreamento em pastagens e na presença do gado, constataram que o método com arame farpado em espiral e uma estaca, foi o mais efetivo e com menor custo. A implementação implicou no acréscimo de $9 \%$ no custo operacional em exploração extensiva de gado de corte, significando uma redução de $27 \%$ no retorno bruto. No entanto, segundo os mesmos autores, acréscimos de $9 \%$ é um custo que dificulta a introdução da prática de arborização em pastagens.

Portanto, o estabelecimento de espécies de leguminosas arbóreas mais adaptadas sem que haja necessidade de proteção de mudas e na presença de animais, poderá reduzir o custo da arborização e permitir a introdução de espécies mesmo em condições de baixa rentabilidade no setor, e em especial para a pecuária extensiva.

Por outro lado, o sucesso da introdução de uma espécie, sem proteção e na presença dos animais, depende do grau de sua aceitabilidade pelos animais (Ash, 1990; Hindrichsen et al., 2004), da velocidade de crescimento e da competição com a pastagem. Segundo McSweeney et al. (2005) e Meirelles et al. (2005), a aceitabilidade da forrageira, pelos animais, pode ser devido ao teor de tanino e também se já é parte de sua dieta na pastagem (Souto, 1967; Souto et al., 1975).

Este trabalho teve por objetivo avaliar o desempenho de 16 espécies de leguminosas arbóreas introduzidas em pastagem estabelecida com capim Tifton-85, a partir de mudas pequenas, sem proteção e na presença de animais.

\section{MATERIAL E MÉTODOS}

Este trabalho foi desenvolvido em pastagem de capim Tifton-85, inserida no Sistema Integrado de Produção Agroecológica (SIPA), na Embrapa Agrobiologia, em Seropédica-RJ (Lat. 21 45'; Long. 4341'; Alt. $33 \mathrm{~m}$ )

O solo na área experimental é um Planossolo Hidromórfico Distrófico Arênico com seguinte composição química: $\mathrm{pH}$ (em água $)=5,6 ; \mathrm{Al}=0,0 \mathrm{cmol} / \mathrm{dm}^{3} ; \mathrm{Ca}+\mathrm{Mg}=2,9$ $\mathrm{cmol} / \mathrm{dm}^{3} ; \mathrm{P}=4 \mathrm{~g} / \mathrm{dm}^{3} ; \mathrm{K}=27 \mathrm{~g} / \mathrm{dm}^{3} ; \mathrm{C}=$ $0,81 \%$ e N $=0,066 \%$.

As 16 espécies de leguminosas arbóreas introduzidas nas pastagem foram as seguintes: 1-Gliricídia (Gliricidia sepium); 2- Albízia (Pseudomanea guachapele); 3Mulungú (Erythrina verna); 4- jurema preta (Mimosa tenuiflora); 5- Sabiá (Mimosa caesalpiniifolia); 6- Angico vermelho (Anadenanthera macrocarpa); 7-Olosericia (Acacia holosericea); 8- Acácia auriculada (Acacia auriculiformis); 9- jurema branca (Mimosa artemisiana); 10- Orelha de negro (Enterolobium contortisiliquum); 11 Guapuruvu (Schizolobium parahyba); 12Mulungú do alto (Erythrina poeppigiana); 13- Coração de negro (Albizia lebbeck); 14Leucena (Leucaena leucocephala); 15Jacarandá bico de pato (Machaerium hirtum); 16- Canafístula (Peltophorum dubium).

As mudas foram produzidas em agosto de 2001 no viveiro do campo experimental da Embrapa Agrobiologia, por meio de sementes inoculadas com estirpes eficientes de rizobio, recomendadas por Faria (2001); também com uma mistura dos fungos micorrízicos, Gigaspora margarita e Glomus clarum; semeadas em saquinhos de 800 à $1000 \mathrm{~g}$ com substrato contendo $30 \%$ de composto orgânico, $30 \%$ de argila, $30 \%$ de areia e $10 \%$ de fosfato de rocha.

O plantio das mudas foi em dezembro de $2001, \log$ após um rebaixamento do pasto pelo gado. Elas foram levadas para o campo com altura entre 40 e $60 \mathrm{~cm}$ (4 a 5 meses de viveiro). O plantio foi feito em covas de 20 $\mathrm{cm} \times 20 \mathrm{~cm} \times 20 \mathrm{~cm}$ de dimensões, adubadas com $100 \mathrm{~g}$ de fosfato de rocha $+10 \mathrm{~g}$ de FTE $\mathrm{Br} 12$ (12\% de $\mathrm{Zn}, 1,6 \%$ de $\mathrm{Cu}, 4 \%$ de $\mathrm{Mn}$ e $1,8 \%$ de B) +25 g de sulfato de potássio +25 g de calcário dolomítico. As covas distan- 
ciadas de 7,5 $\mathrm{m}$ foram feitas manualmente com enxadões. Foram plantados oito indivíduos de cada espécie em uma área experimental de 0,9 ha.

O sistema de pastejo foi o rotacionado, conforme preconizado no Sistema Integrado de Produção Agroecológica (SIPA), não ultrapassando cinco dias de ocupação através da estimativa visual de disponibilidade de forragem ( $2300 \mathrm{~kg}$ de $\mathrm{MS} / \mathrm{ha})$. Dessa forma, manteve-se, a longevidade e produtividade da pastagem e o fornecimento de alimento em quantidade e qualidade atendendo às exigências nutricionais dos animais (Silva et al., 1998), assim contribuindo para o estabelecimento das mudas das leguminosas nas pastagens.

Foram feitas três avaliações, usando lotação de 30 novilhas mestiças leiteiras/ha, representativas da região, com peso vivo em torno de $300 \mathrm{~kg} /$ animal. Os animais foram colocados nos piquetes, quando o pasto apresentava boa disponibilidade de forragem com capacidade para receber 30 novilhas sem o risco de altas pressões de pastejo nos períodos de avaliação. $1^{\mathrm{a}}$ avaliação foi feita no período de 06/05/2002 a 08/05/ 2002 (período com temperatura média $24,0^{\circ} \mathrm{C}$ e precipitação de $8,7 \mathrm{~mm}$ ); a $2^{\mathrm{a}}$ avaliação de 29/07/2002 a 03/08/2002 (período com temperatura média $20,4^{\circ} \mathrm{C}$ e precipitação de 14,0 mm); e a $3^{\text {a }}$ avaliação de 08/09/2002 a 10/09/ 2002 (período com temperatura média $19,3^{\circ} \mathrm{C}$ e precipitação de 12,0 mm).

Antes e depois de cada avaliação foram feitas contagem do número de brotos e a medição do comprimento dos brotos das mudas.

As variáveis estudadas foram as seguintes: X1- comprimento dos brotos antes do pastejo animal; X2- comprimento dos brotos após o pastejo; X3- diferenças no comprimento dos brotos com o pastejo; $\mathrm{X} 4$ número de brotos na muda antes do pastejo; X5- número de brotos na muda após o pastejo; X6- diferença no número de brotos com o pastejo; X7- relação entre o comprimento e o número de brotos com o pastejo.
O experimento teve 16 tratamentos (16 espécies arbóreas) em blocos inteiramente casualizados com oito repetições (oito mudas de cada espécie) em três períodos de avaliação. Considerou-se, segundo Pimentel Gomes (2000) como experimento em parcela subdivididas, uma vez que, as avaliações sempre foram realizadas nas mesmas parcelas. A correlação de Pearson entre as variáveis foi avaliada pelo Software SAEG 9.0 (2005). As diferenças dos vetores das médias de tratamentos foram verificadas por variância multivariada Manova, usando os testes de Hotelling-Lawley, Pillai, Wilks e Roy (Ribeiro Junior, 2001), para testar a hipótese de nulidade, que é a igualdade entre os vetores de médias dos tratamentos. Com base nos resultados das correlações de Pearson, significativas entre as variáveis (tabela I), e na hipótese de nulidade rejeitada pelos quatro testes de Manova, a análise dos dados do experimento foi feita por meio da análise de variância multivariada, segundo Pimentel Gomes (2000) e Ribeiro Junior

Tabela I. Correlações significativas entre variáveis. (Significants correlation among variables).

\begin{tabular}{cccc}
\hline Variável & Variável & $r$ & Significâção de $r$ \\
\hline X1 & X2 & 0,99 & $0,0001^{* *}$ \\
X1 & X4 & 0,98 & $0,0001^{* *}$ \\
X1 & X5 & 0,98 & $0,0001^{* *}$ \\
X2 & X4 & 0,98 & $0,0001^{* *}$ \\
X2 & X5 & 0,98 & $0,0001^{* *}$ \\
X3 & X6 & 0,74 & $0,0001^{* *}$ \\
X3 & X7 & 0,76 & $0,0333^{*}$ \\
X4 & X6 & 0,99 & $0,0001^{* *}$ \\
\hline
\end{tabular}

$\mathrm{X} 1$ : comprimento dos brotos antes do pastejo; $\mathrm{X} 2$ : comprimento dos brotos após o pastejo; X3: diferenças no comprimento dos brotos com o pastejo; X4: número de brotos na muda antes do pastejo; X5: número de brotos na muda após o pastejo; X6: diferença no número de brotos com o pastejo; X7: relação entre o comprimento dos brotos e o número de brotos com o pastejo. ${ }^{*} p<0,05 ;{ }^{* *} p<0,01$. 


\section{DIAS, SOUTO E FRANCO}

(2001). Para comparar as médias dos tratamentos, duas a duas, para cada variável, usou-se os intervalos de confiança simultâneos de Bonferroni (Ferreira 2003).

\section{RESULTADOEDISCUSSÃO}

As diferenças entre as médias dos tratamentos para cada variável, considerando a influência das demais variáveis são mostradas na tabela II.

As variáveis X1 e X4 quando comparadas, respectivamente, com X2 e X5, foram descartadas, segundo Ribeiro Junior (2001), por apresentarem maiores valores de r (tabela I) e menores para a estatística F.

O maior comprimento dos brotos após o pastejo (variável X2) foi encontrado para a jurema preta na $3^{\mathrm{a}}$ avaliação (tratamento 12 ), mostrando que esta espécie teve baixa aceitabilidade pelos animais. Esta baixa aceitabilidade não foi devido à presença de acúleos na planta, uma vez que, outras leguminosas testadas também apresentavam acúleos, como a sabiá, jurema branca e mulungú do alto, e foram pastejadas.

$\mathrm{Na}$ avaliação da aceitabilidade das plantas é muito importante também ser levado em conta a preferência dos animais no pasto, se determinada planta já fez parte de sua dieta (Souto, 1967; Souto et al., 1975). Os animais usados no presente trabalho não tiveram em sua dieta, anteriormente, as leguminosas usadas neste experimento.

A maior diferença no comprimento dos brotos com o pastejo (variável X3), dada pela diferença no comprimento de brotos medida antes e após o pastejo, foi para a acácia auriculada na $3^{\mathrm{a}}$ avaliação (tratamento 24) que não diferenciou significativamente de gliricídia na $1^{\mathrm{a}}$ avaliação (tratamentos 1 ), de acácia auriculada na $2^{\mathrm{a}}$ avaliação (tratamento 23) e de leucena na $1^{\text {a }}$ avaliação (tratamentos 40), mostrando que estas leguminosas foram as mais consumidas pelos animais (tabela II).

Dessas leguminosas, a gliricídia e a leucena, por serem palatáveis (Franco e
Souto, 1986; Souto et al., 1992; Dias et al., 2004), e apresentarem níveis baixos de tanino (Hindrichsen et al., 2004; McSweeney et al., 2005), são recomendadas para bancos de proteína (Rangel et al., 2001; Zoby, 2001). Entretanto a gliricídia foi considerada por Mendonça (2005) como de baixa palatabilidade como forragem verde, devido à relutância dos animais em consumi-la, exigindo por isso um período de adaptação a dieta. O mesmo autor salienta que sob a forma de feno ela foi melhor aceita pelo gado. No entanto, Souto et al., 1992, confirmaram que a baixa palatabilidade de gliricídia dependeu do acesso usado sob certas condições.

Uma das menores diferenças no comprimento dos brotos com o pastejo (variável X3), em relação as espécies que apresentaram maiores comprimento de broto $(5942 \mathrm{~cm} /$ planta) antes do pastejo (variável X1), foi observada para jurema preta na $3^{\mathrm{a}}$ avaliação - tratamento 12 (tabela II).

A maior quantidade de brotos após o pastejo (variável X5) foi registrada para jurema preta (tratamento 12), com 193 brotos/ planta (tabela II).

A leucena na $3^{a}$ avaliação (tratamento 41), a gliricídia na $2^{\mathrm{a}}$ avaliação (tratamento 2) e acácia auriculada na $3^{\mathrm{a}}$ avaliação (tratamento 24), apresentaram as maiores diferenças no número de brotos após o pastejo (variável X6). Pelos resultados das variáveis X3 (diferença no comprimento dos brotos com o pastejo) e X7 (relação entre comprimento e número de brotos com o pastejo), a leucena, gliricídia e a acácia auriculada são as menos indicadas para serem introduzidas nas pastagens da região, sem a proteção das mudas e na presença dos animais (tabela II).

A jurema preta, como observado anteriormente, mostrou também a menor diferença no comprimento (variável X3) e número (variável X6) de brotos com pastejo, entre as espécies com maiores valores no comprimento e número de brotos antes do pastejo (tabela II), consolidando assim a 
Tabela II. Efeitos dos tratamentos no comprimento e número de brotos de leguminosas arbóreas, introduzidas em pastagem de Tifton-85, sem proteção das mudas e na presença de animais. Médias de oito repetições. (Effects of the treatments on the sproutings length and numbers of legume trees introduced during establishment of Tifton-85 grass without protection for young plants and in the presence of animals. Means of eight repetitions).

\begin{tabular}{|c|c|c|c|c|c|c|c|c|c|}
\hline \multirow[b]{2}{*}{ Tratamento } & \multirow[b]{2}{*}{ Leguminosa } & \multirow[b]{2}{*}{ Avaliação $^{1}$} & \multicolumn{7}{|c|}{ Variáveis $^{2}$} \\
\hline & & & $\begin{array}{l}\mathrm{X} 1 \\
\mathrm{~cm}\end{array}$ & $\begin{array}{l}\mathrm{X} 2 \\
\mathrm{~cm}\end{array}$ & $\begin{array}{l}X 3 \\
\mathrm{~cm}\end{array}$ & $\begin{array}{l}\mathrm{X} 4 \\
\mathrm{n}^{\circ}\end{array}$ & $\begin{array}{l}X 5 \\
n^{\circ}\end{array}$ & $\begin{array}{l}x 6 \\
n^{\circ}\end{array}$ & $\begin{array}{c}X 7 \\
\mathrm{~cm} / \mathrm{n}^{\circ}\end{array}$ \\
\hline 1 & Gliricídia & 1 & 312 & $231^{\mathrm{b}}$ & $80^{\mathrm{ab}}$ & 5 & $4^{b}$ & $1^{\mathrm{b}}$ & $58^{a}$ \\
\hline 2 & & 2 & 364 & $305^{b}$ & $59^{\mathrm{bc}}$ & 23 & $16^{b}$ & $7^{a}$ & $19^{c}$ \\
\hline 3 & & 3 & 647 & $621^{\mathrm{b}}$ & $26^{c}$ & 26 & $24^{\mathrm{b}}$ & $2^{b}$ & $26^{b c}$ \\
\hline 4 & Albízia & 1 & 9 & $7^{\mathrm{b}}$ & $2^{c}$ & 2 & $1^{b}$ & $1^{\mathrm{b}}$ & $7^{\text {cd }}$ \\
\hline 5 & & 2 & 18 & $17^{\mathrm{b}}$ & $1^{c}$ & 2 & $1^{b}$ & $1^{\mathrm{b}}$ & $17^{\mathrm{dd}}$ \\
\hline 6 & & 3 & 89 & $67^{\mathrm{b}}$ & $22^{c}$ & 3 & $2^{b}$ & $1^{\mathrm{b}}$ & $34^{b}$ \\
\hline 7 & Mulungú & 1 & 2 & $1^{\mathrm{b}}$ & $1^{c}$ & 2 & $1^{b}$ & $1^{\mathrm{b}}$ & $1^{\mathrm{d}}$ \\
\hline 8 & & 2 & 2 & $1^{b}$ & $1^{c}$ & 2 & $1^{b}$ & $1^{\mathrm{b}}$ & $1^{\mathrm{d}}$ \\
\hline 9 & & 3 & 4 & $3^{b}$ & $1^{c}$ & 2 & $1^{b}$ & $1^{\mathrm{b}}$ & $3^{d}$ \\
\hline 10 & Jurema preta & 1 & 976 & $975^{\mathrm{b}}$ & $1^{c}$ & 28 & $27^{\mathrm{b}}$ & $1^{\mathrm{b}}$ & $36^{b}$ \\
\hline 11 & & 2 & 2567 & $2566^{b}$ & $1^{c}$ & 81 & $80^{\mathrm{b}}$ & $1^{\mathrm{b}}$ & $32^{\mathrm{b}}$ \\
\hline 12 & & 3 & 5942 & $5941^{a}$ & $1^{c}$ & 194 & $193^{a}$ & $1^{\mathrm{b}}$ & 31 \\
\hline 13 & Sabiá & 1 & 302 & $261^{b}$ & $41^{\mathrm{bc}}$ & 7 & $6^{b}$ & $1^{\mathrm{b}}$ & $44^{\mathrm{ab}}$ \\
\hline 14 & & 2 & 413 & $393^{b}$ & $20^{c}$ & 14 & $11^{\mathrm{b}}$ & $3^{b}$ & $36^{b}$ \\
\hline 15 & & 3 & 899 & $884^{\mathrm{b}}$ & $15^{c}$ & 43 & $41^{\mathrm{b}}$ & $2^{b}$ & $22^{b c}$ \\
\hline 16 & Angico vermelho & 1 & 22 & $15^{\mathrm{b}}$ & $7^{c}$ & 3 & $2^{b}$ & $1^{\mathrm{b}}$ & $8^{\text {cd }}$ \\
\hline 17 & & 2 & 14 & $6^{b}$ & $8^{c}$ & 2 & $1^{b}$ & $1^{\mathrm{b}}$ & $6^{\mathrm{cd}}$ \\
\hline 18 & & 3 & 41 & $40^{\mathrm{b}}$ & $1^{c}$ & 3 & $2^{b}$ & $1^{\mathrm{b}}$ & $20^{c}$ \\
\hline 19 & Oloserícia & 1 & 7 & $5^{b}$ & $2^{c}$ & 2 & $1^{b}$ & $1^{\mathrm{b}}$ & $5^{c}$ \\
\hline 20 & & 2 & 94 & $93^{b}$ & $1^{c}$ & 3 & $2^{b}$ & $1^{\mathrm{b}}$ & $47^{\mathrm{ab}}$ \\
\hline 21 & & 3 & 261 & $259^{b}$ & $2^{c}$ & 7 & $6^{b}$ & $1^{\mathrm{b}}$ & $43^{\mathrm{ab}}$ \\
\hline 22 & Acácia auriculada & 1 & 70 & $59^{b}$ & $11^{\mathrm{c}}$ & 5 & $4^{b}$ & $1^{\mathrm{b}}$ & $15^{\mathrm{dd}}$ \\
\hline 23 & & 2 & 244 & $164^{\mathrm{b}}$ & $80^{\mathrm{ab}}$ & 12 & $8^{b}$ & $4^{b}$ & $21^{c}$ \\
\hline 24 & & 3 & 404 & $239^{b}$ & $165^{a}$ & 24 & $16^{b}$ & $8^{a}$ & $15^{\mathrm{cd}}$ \\
\hline 25 & Jurema branca & 1 & 76 & $75^{\mathrm{b}}$ & $1^{c}$ & 5 & $4^{b}$ & $1^{\mathrm{b}}$ & $19^{c}$ \\
\hline 26 & & 2 & 168 & $167^{b}$ & $1^{c}$ & 8 & $7^{\mathrm{b}}$ & $1^{\mathrm{b}}$ & $24^{\text {bc }}$ \\
\hline 27 & & 3 & 438 & $415^{\mathrm{b}}$ & $23^{c}$ & 21 & $19^{b}$ & $2^{\mathrm{b}}$ & $22^{c}$ \\
\hline 28 & Orelha de negro & 1 & 34 & $31^{\mathrm{b}}$ & $3^{c}$ & 2 & $1^{\mathrm{bb}}$ & $1^{\mathrm{b}}$ & $31^{b}$ \\
\hline 29 & & 2 & 22 & $21^{\mathrm{b}}$ & $1^{c}$ & 2 & $1^{b}$ & $1^{\mathrm{b}}$ & $21^{c}$ \\
\hline 30 & & 3 & 83 & $75^{\mathrm{b}}$ & $8^{c}$ & 4 & $2^{b}$ & $2^{b}$ & $38^{b}$ \\
\hline 31 & Guapuruvu & 1 & 7 & $6^{b}$ & $1^{c}$ & 2 & $1^{b}$ & $1^{\mathrm{b}}$ & $6^{c}$ \\
\hline 32 & & 2 & 2 & $1^{\mathrm{b}}$ & $1^{c}$ & 2 & $1^{b}$ & $1^{\mathrm{b}}$ & $1^{c}$ \\
\hline 33 & & 3 & 3 & $1^{b}$ & $2^{c}$ & 2 & $1^{b}$ & $1^{\mathrm{b}}$ & $1^{c}$ \\
\hline 34 & Mulungú do alto & 1 & 45 & $30^{b}$ & $15^{c}$ & 3 & $2^{\mathrm{b}}$ & $1^{\mathrm{b}}$ & $15^{\mathrm{cd}}$ \\
\hline 35 & & 2 & 93 & $34^{b}$ & $59^{b c}$ & 5 & $1^{b}$ & $4^{b}$ & $34^{b}$ \\
\hline 36 & & 3 & 66 & $52^{b}$ & $14^{c}$ & 5 & $3^{b}$ & $2^{\mathrm{b}}$ & $17^{\mathrm{dd}}$ \\
\hline 37 & Coração de negro & 1 & 19 & $14^{\mathrm{b}}$ & $5^{c}$ & 3 & $2^{\mathrm{b}}$ & $1^{\mathrm{b}}$ & $7^{c}$ \\
\hline 38 & & 2 & 7 & $5^{b}$ & $2^{c}$ & 2 & $1^{\mathrm{b}}$ & $1^{\mathrm{b}}$ & $5^{c}$ \\
\hline 39 & & 3 & 12 & $10^{\mathrm{b}}$ & $2^{c}$ & 2 & $1^{b}$ & $1^{\mathrm{b}}$ & 10 \\
\hline 40 & Leucena & 1 & 226 & $112^{\mathrm{b}}$ & $114^{\mathrm{ab}}$ & 8 & $7^{b}$ & $1^{\mathrm{b}}$ & 16 \\
\hline 41 & & 2 & 237 & 163 & $74^{\mathrm{bc}}$ & 16 & $8^{b}$ & $8^{\mathrm{a}}$ & 20 \\
\hline 42 & & 3 & 385 & $329^{b}$ & $56^{\mathrm{bc}}$ & 25 & $23^{b}$ & $2^{b}$ & $14^{\mathrm{d}}$ \\
\hline
\end{tabular}


Tabela II (continuação). Efeitos dos tratamentos no comprimento e número de brotos de leguminosas arbóreas, introduzidas em pastagem de Tifton-85, sem proteção das mudas e na presença de animais. Médias de oito repetições. (Effects of the treatments on the sproutings length and numbers of legume trees introduced during establishment of Tifton- 85 grass without protection for young plants and in the presence of animals. Means of eight repetitions).

\begin{tabular}{|c|c|c|c|c|c|c|c|c|c|}
\hline \multirow[b]{2}{*}{ Tratamento } & \multirow[b]{2}{*}{ Leguminosa } & \multirow[b]{2}{*}{ Avaliação ${ }^{1}$} & \multicolumn{7}{|c|}{ Variáveis $^{2}$} \\
\hline & & & $\begin{array}{l}\mathrm{X} 1 \\
\mathrm{~cm}\end{array}$ & $\begin{array}{l}\mathrm{X} 2 \\
\mathrm{~cm}\end{array}$ & $\begin{array}{l}\text { X3 } \\
\mathrm{cm}\end{array}$ & $\begin{array}{l}X 4 \\
n^{\circ}\end{array}$ & $\begin{array}{l}X 5 \\
n^{\circ}\end{array}$ & $\begin{array}{l}\mathrm{X} 6 \\
\mathrm{n}^{\circ}\end{array}$ & $\begin{array}{c}X 7 \\
\mathrm{~cm} / \mathrm{n}^{\circ}\end{array}$ \\
\hline 43 & Jacarandá bico de pato & 1 & 36 & $29^{b}$ & $7^{c}$ & 4 & $3^{b}$ & $1^{b}$ & $10^{\text {cd }}$ \\
\hline 44 & & 2 & 58 & $56^{b}$ & $2^{c}$ & 3 & $2^{\mathrm{b}}$ & $1^{b}$ & $28^{\mathrm{bc}}$ \\
\hline 45 & & 3 & 110 & $99^{b}$ & $11^{c}$ & 6 & $5^{b}$ & $1^{b}$ & $20^{c}$ \\
\hline 46 & Canafístula & 1 & 2 & $1^{b}$ & $1^{c}$ & 2 & $1^{\mathrm{b}}$ & $1^{\mathrm{b}}$ & $1^{\mathrm{d}}$ \\
\hline 47 & & 2 & 7 & $6^{b}$ & $1^{c}$ & 2 & $1^{\mathrm{b}}$ & $1^{b}$ & $6^{c}$ \\
\hline 48 & & 3 & 31 & $30^{\mathrm{b}}$ & $1^{c}$ & 2 & $1^{\mathrm{b}}$ & $1^{\mathrm{b}}$ & $30^{b}$ \\
\hline
\end{tabular}

11: 06/05/2002 a 09//05/2002; 2: 29/07/2002 a 03/08/2002; 3: 08/09/2002 a 10/09/2002.

${ }^{2}$ X1: comprimento dos brotos antes do pastejo; X2: comprimento dos brotos após o pastejo; X3: diferenças no comprimento dos brotos com o pastejo; X4: número de brotos na muda antes do pastejo; X5: número de brotos na muda após o pastejo; X6: diferenças no número de brotos com o pastejo; X7: relação X2/X5.

a,b,c,dMédias seguidas por letras iguais na coluna, não diferem entre si, por meio dos intervalos de confiança de Bonferroni, a $5 \%$ de probabilidade.

sua indicação para a região, como a leguminosa com maior perspectiva de sucesso, do ponto de vista ecológico, se as mudas forem introduzidas na pastagem, sem proteção e na presença dos animais. Dias et al. (2005), trabalhando com as mesmas 16 espécies do presente trabalho, incluíram a jurema preta no grupo de espécies que apresentou o maior número de plantas sobreviventes e menos pastejadas. Todavia, muitas forrageiras usadas na alimentação de ruminantes, possuem alto teor de proteína bruta (16\%), como a jurema preta, mas apresentam baixa digestibilidade, pois apresentam altos níveis de tanino (122 g/ kg MS), (Nozella, 2001).

Os maiores valores para a relação entre comprimento e número de brotos com o

\section{BIBLIOGRAFIA}

Andrade, C.M.S., J.F. Valentim e J.C. Carneiro. 2002. Árvores de Baginha (Stryphnodendron guianensis) em ecossistemas de pastagens cultivadas na Amazonia Ocidental. Rev. Bras. pastejo (variável X7), foram registradas para Gliricídia (tratamento 1) e Sabiá (tratamento 13), mostrando que o número de brotos dessas espécies foi mais afetado durante o pastejo do que o seu comprimento.

\section{CONCLUSÃO}

Das leguminosas testadas, a jurema preta, apesar da baixa aceitabilidade pelos animais, é a indicada para ser introduzida com sucesso, do ponto de vista ecológico nas pastagens de capim Tifton-85 da região, sem a proteção de suas mudas e na presença do gado, beneficiando também o solo e as forrageiras sob sua copa, assim como os animais, com a sua sombra.

Zootecn., 31: 1-5.

Ash, A.J. 1990. The effect of supplementation with leaves from the leguminous trees Sesbania grandiflora, Albizia chinensis and Gliricidia 


\section{MUDAS DE LEGUMINOSAS ARBÓREAS EM PASTAGEM DE TIFTON-85 COM ANIMAIS}

sepium on the intake digestibility of guinea grass hay by goats. Anim. Feed Sci. Tech., 28: 225-232.

Costa, N.L., J.A. Magalhães, C.R. Towsend e R.G.A. Pereira. 2005. Produtividade de leguminosas forrageiras sob sombreamento de eucalípto. 2005. Disponível em: <http// www.boletimpecuario.com.br/artigos> Acesso em nov/2005.

Dias, P.F. 2005. Importância da arborização de pastagens com leguminosas fixadoras de nitrogênio. 2005. Tese (Doutorado em Fitotecnia). Universidade Federal Rural do Rio de Janeiro. Seropédica. $140 \mathrm{p}$

Dias, P.F., S.M. Souto e A.A. Franco. 2005. Introdução e avaliação de leguminosas arbóreas em pastagens da baixada e região serrana do estado do Rio de Janeiro. Embrapa Agrobiologia. Boletim de Pesquisa e Desenvolvimento, 09. Seropédica: Embrapa Agrobiologia, 2005. 24 p.

Dias, P.F., S.M. Souto, B.M. Pereira, R.S. Lizieire, A.M. Zanine, L.T. Schimidt e A.A. Franco. 2004. Sobrevivência de estacas de gliricídia (Gliricidia sepium) como moirão vivo. Past. Trop., 26: 55-62.

Faria, S.M. 2001. Obtenção de estirpes de rizóbio eficientes na fixação de nitrogênio para espécies florestais (aproximação 2001). Embrapa Agrobiologia. Boletim de Pesquisa e Desenvolvimento, 09. Embrapa Agrobiologia. Seropédica. $21 \mathrm{p}$.

Ferreira, D.E. 2003. Análise de variância multivariada. Disponível em <http://www. dex.ufla.br/daniel>. Acesso em nov/2005.

Franco, A. e S.M. Souto. 1986. Leucaena leucocephala- uma leguminosa com múltiplas utilidades para os trópicos. CNPAB, Comunicado Técnico $n^{\circ}$ 2. CNPAB. Seropédica. $7 p$.

Hindrichsen, I.K., P.O. Osuji, A.A. Odenyo, J. Madsen and T. Hvelplund. 2004. Effect of supplementation of maize stover with foliage of varios tropical multipurpose trees and Lablab purpureus on intake, rumen fermentation, digesta kinetics and microbial protein supply of sheep. Anim. Feed Sci. Tech., 113: 83-96.

Ibrahim, M., A. Schlonvoiggt, C. Camargo and M. Souza. 2001. Multistrata silvopastoral systems for increasing productivity and conservation of natural resources in Central America. In:
International Grassland Congress, 19. 2001. Brasília. Proceedings... EMBRAPA. Brasília. p. 645-649.

Kanninen, M. 2001. Sistemas silvopastoriles y almacenamiento de carbono: potential para América Latina (en línea). Plataforma Electrónica sobre Ganaderia y Medio Ambiente, LEAD/FAO/CATIE. Disponible En: <http:// lead.virtualcentre.org/es/ele/conferencia3/ articulo.htm>. Acesso em jul. 2001.

McAdam, J., M.R. Mosquera-Losada, V. Papanastasis, A. Pardini and A. Rigueiro-Rodrigues. 2005. Silvopastoral systems: analysis of an alternative to open swards. In: XX International Grassland Congress, 20. 2005. Dublin. Proceedings... IGC. Dublin.. p. 758.

McSweeney, C.S., J. Gough, L.L. Conlan, M.P. Hegarty, B. Palmer and D.O. Krause. 2005. Nutritive value assessment of the tropical shrub legume Acacia angustissima: anti-nutritional compounds and in vitro digestibility. Anim. Feed Sci. Tech., 121: 175-190.

Meirelles, P.R.L., L.A.R. Batistas, G.B. Souza, B. Lempp e C. Costa. 2005. Quantificação e distribuição de taninos em gramíneas forrageiras tropicais. Em: Reunião Anual da Sociedade Brasileira de Zootecnia, 2005. Goiânia....SBZ. Goiânia.

Mendonça, J.F.B. 2005. Gliricidia: a planta de multi-propósito para agricultura tropical. Disponível em: <http://www.boletimpecuario. com.br/artigos>. Acesso em 02/2005.

Montoya, L.J. e A.J. Baggio. 1991. Estudos econômicos da introdução de mudas altas para sombreamento de pastagens. Em: Encontro Brasileiro de Economia e Planejamento Florestal, 1. 1991. Curitiba: Anais... Embrapa Floresta. Colombo. 2: 172-191.

Naranjo, L. 2000. Sistemas agroforestales para la producción pecuaria y la conservación de la biodiversidad (en línea). Disponible en: <http:// lead.virtualcentre.org/es/ele/conferencia2/vbconfe18.htm>. Acesso em: out. 2000.

Nozella, E.F. 2001. Determinação de taninos em plantas com potencial forrageiro para ruminantes.Tese (Mestrado). Universidade de São Paulo. Piracicaba.

Pimentel-Gomes, F. 2000. Curso de estatística experimental. 22 ed. Piracicaba: ESALQ/USP, $363 \mathrm{p}$.

Archivos de zootecnia vol. 58, núm. 222, p. 209. 


\section{DIAS, SOUTO E FRANCO}

Rangel, J.H.A., O.M. Carvalho Filho e S.A. Almeida. 2001. Experiências com o uso da Gliricidia sepíum na alimentação animal nop nordeste brasileiro. Em: Carvalho, M.M., Alvim, M.J. e Carneiro, J.C. (Eds). Sistemas agroflorestais pecuários: Opções de sustentabilidade para as áreas tropicais e subtropicais. Embrapa Gado de Leite. Juiz de Fora. FAO. Brasília. p. 35-44.

Ribeiro Junior, J.I. 2001. Análises estatísticas no SAEG. UFV. Viçosa. $250 \mathrm{p}$.

SAEG 9.0. 2005. Sistema para análises estatísticas-SAEG. Versão 9.0. Fundação Arthur Bernardes. Viçosa.

Silva, S.C. da, M.M. Passanezi, R.A. Comevalli, C.G. Pedreira e J.L.M. Fagundes. 1998. Bases para o estabelecimento do manejo de Cynodon sp. para o pastejo e conservação. Em: Peixoto, A.M., Moura, J.C. e Faria, V.P. (Eds). Manejo de pastagens de Tifton, Coastcross e Estrela. FEALQ. Piracicaba. p. 129-150.
Souto, S.M. 1967. Aceitabilidade e persistência de forrageiras tropicais. Em: Reunião da Sociedade Brasileira de Zootecnia, 7. 1967. Piracicaba. Anais... SBZ. Piracicaba. p. 11-14.

Souto, S.M., H. de Polli, D.L. Almeida, F.F. Duque, R.L. Assis e P.A. Eiras. 1992. Outros usos de leguminosas convencionalmente utilizadas par adubo verde. CNPAB, Documentos $n^{\circ} 11$. CNPAB. Seropédica. $39 \mathrm{p}$.

Souto, S.M., C.R. Lima e E.D. Lucas. 1975 Palatabilidade de leguminosas forrageiras. Pesqui. Agropecu. Bras., 10: 7-11.

Zoby, J.L.F. 2001. Leucena em banco de proteína como complemento de pastagens do cerrado na alimentação de bovinos. Em: Carvalho, M.M., Alvim, M.J. e Carneiro, J.C. (Eds). Sistemas agroflorestais pecuários: Opções de sustentabilidade para as areas tropicais e subtropicais. 2001. Embrapa Gado de Leite. Juiz de Fora. FAO. Juiz de Fora. p. 45-49. 